\title{
Un modelo para la enseñanza de las matemáticas en secundaria
}

DOI: https://doi.org/10.32870/dse.v0i10.286

\section{Luis Felipe Gómez López* \\ Juan Carlos Silas Casillas** \\ Eduardo Miranda Montoya***}

\begin{abstract}
Resumen: En este artículo se presenta un modelo para la enseñanza de las matemáticas en secundaria formulado a partir de dos conjuntos de insumos: a) las necesidades de mejora identificadas mediante la observación de clases de primer grado, de la revisión del contenido de foros virtuales en que participaron 30 docentes y las bitácoras que escribieron en el lapso de un año; b) la literatura especializada y la investigación acerca de la enseñanza eficaz de las matemáticas en la educación secundaria. El modelo contiene tres dimensiones centrales: la enseñanza, el aprendizaje y la evaluación, así como dos ejes transversales: la matematización de la realidad y los aprendizajes esperados. ITESO. Palabras clave: Sistema de enseñanza, educación general básica, matemáticas.
\end{abstract}

Abstract: This paper presents a model for teaching mathematics in junior high school. it is formulated from two sets of inputs: a) the need for improvement identified by observing 7th grade classes, the review the contents of the participation of teachers in virtual forums and blogs written by 30 of them in the span of a year; $b$ ) the literature and research on effective teaching of mathematics in junior high school. The model contains three central dimensions: teaching, learning and assessment, as well as two cross-cutting themes: the mathematization of reality and the expected learning outcomes. Keywords: System of education, primary education, mathematics.

\section{Introducción}

En una investigación sobre enseñanza de las matemáticas en secundaria llevada a cabo entre los años 2012 y 2013 que tenía como finalidad "Determinar la efectividad de una intervención basada en el diseño de estrategias y escenarios educativos enfocados en el desarrollo de competencias matemáticas en un ambiente que favorezca la convivencia respetuosa entre los alumnos de secundaria", se analizaron los obstáculos para que los alumnos desarrollen las competencias que prescribe el currículo escolar.

* Doctor en educación por la Universidad Autónoma de Aguascalientes. Profesor/investigador del Instituto Tecnológico y de Estudios Superiores de Occidente (ITESO), en donde coordina el Doctorado Interinstitucional en Educación. Cuenta con diversas publicaciones sobre el tema de procesos de aprendizaje. Correo electrónico: 1gomez@iteso.mx

** Licenciado en psicología educativa y magíster en educación por la Universidad del Valle de Atemajac en Guadalajara, Jalisco, Mexico. Doctorado en Educational Policy and Leadership (2000) por la Universidad de Kansas, Estados Unidos. Actualmente se desempeña como profesor investigador de tiempo completo en el Departamento de Educación y Valores, en el ITESO. Correo electrónico: silasji@iteso.mx

***Doctor en matemáticas, con la especialidad de Matemática Educativa por el Centro de Investigación y de Estudios Avanzados (Cinvestav) del Instituto Politécnico Nacional. Profesor/investigador en el departamento de Física y Matemáticas, coordinador del área de Cálculo, en el ITESO. Cuenta con publicaciones en el área de Matemática Educativa. Correo electrónico: emiranda@, iteso.mx 
Estas dificultades fueron identificadas a partir de la observación de 26 clases de primer grado de secundaria, de la revisión del contenido de 35 foros virtuales en que participaron 30 profesores de ese grado y de las bitácoras que escribieron durante el lapso de un año. Los resultados de la investigación se encuentran en otras publicaciones. Aquí sólo se presentan, de manera sintética las dificultades, que se toman como necesidades de mejora y a partir de las cuales se propone un modelo que contribuya a la mejora de la enseñanza de las matemáticas en secundaria. El contexto de donde surgió la información fueron secundarias públicas, pero muy probablemente sirva por igual para la educación privada.

En la investigación mencionada se encontró que algunos de los profesores no tienen una comprensión profunda de los temas que enseñan, lo que dificulta que diseñen variadas maneras de ayudar a los alumnos a construir el conocimiento. En ocasiones ellos mismos causan confusión a sus estudiantes respecto a temas como números negativos y multiplicación de fracciones. A otros profesores, les hacen falta conocimientos pedagógicos que les permitan diseñar actividades de aprendizaje apropiadas. También se notó la falta de didácticas específicas, pues las disciplinas muy codificadas como las matemáticas, se centran más en didácticas específicas, que otras disciplinas menos codificadas, como las que engloban las ciencias sociales (Smeby, 1996). Un profesor debe tener tanto el contenido disciplinal como el pedagógico, pues como señala Libâneo (2014) no puede haber una separación entre la didáctica, la epistemología de la disciplina y la metodología de la enseñanza.

Por otra parte, muchos profesores desconocen el modelo de competencias de la Secretaría de Educación Pública (SEP), aunque pueden repetir los términos, no muestran una comprensión conceptual, ni se observó que lo tomaran en cuenta durante el trabajo en las aulas. Probablemente el desconocimiento se deba a que

Las innovaciones que se introducen en el currículo procede, en primera instancia, del mandato de la autoridad educativa (estrategia "de arriba hacia abajo" y de "afuera hacia adentro") y la autoría de orden intelectual de los modelos de cambio proviene de los expertos en contenido o de los diseñadores curriculares y asesores, por lo que en muchos casos se sigue trabajando en una lógica de implantación unidireccional o incluso imposición de las autoridades o especialistas hacia los actores (profesores y alumnos) (Díaz Barriga, 2012: 34).

De lo afirmado por Díaz Barriga se desprende que en ocasiones se limita al profesor a desempeñar un papel técnico como ejecutor de cursos diseñados por la autoridad central, que les proporciona el programa completo del año, la división por bimestres, los aprendizajes esperados, las maneras específicas de llevar a cabo cada clase y los recursos que pueden utilizar.

Probablemente a eso se deba también que los profesores no se centran en que los alumnos aprendan, sino en seguir el programa que se les ha prescrito y lo hacen de manera episódica; es decir, enseñan un tema y se olvidan de él para continuar con el siguiente que aparece en el progra- 
ma. El profesor termina el programa, pero el alumno no obtuvo los aprendizajes esperados. Como se trata de seguir un programa y no de lograr que el alumno aprenda muchos de los profesores no elaboran un diagnóstico inicial, a menos que se los indique la autoridad.

Un aspecto muy importante que no favorece el aprendizaje es que los profesores confunden la evaluación con la calificación. Califican aspectos no relacionados con el aprendizaje como limpieza, asistencia, tarea, conducta, trabajo en clase, etc. Aunque está bien evaluar esos aspectos como parte del proceso formativo, el que los alumnos obtengan una calificación aprobatoria por tener una buena conducta y haber asistido a clases sin aprender, distorsiona el proceso formativo del alumno y se convierte en un efecto perverso, pues los alumnos aprueban los cursos sin haber construido el mínimo de aprendizajes esperados. Las evaluaciones nacionales (INEE, 2012) e internacionales (OCDE, 2013) así lo demuestran.

Los profesores parten del supuesto que los alumnos deben de llegar a la secundaria con interés por aprender lo que en la escuela les quieran enseñar y les desmotiva que no sea así, en lugar de buscar estrategias para generar la involucración e interés de los alumnos. Todas estas dificultades pueden verse de manera sintética en la siguiente tabla.

\section{Tabla 1. Las dificultades para la enseñanza eficaz de las matemáticas}

\begin{tabular}{|l|l|l|l|}
\hline \multicolumn{1}{|c|}{ Desconocimiento } & \multicolumn{1}{|c|}{ Motivación } & \multicolumn{1}{|c|}{ Evaluación } & \multicolumn{1}{|c|}{ Enseñanza } \\
\hline Desconocimiento de la & $\begin{array}{l}\text { Ausencia de estra- } \\
\text { tegias para buscar }\end{array}$ & $\begin{array}{l}\text { Confunden la eva- } \\
\text { luación del apren- } \\
\text { dizaje con la cali- }\end{array}$ & $\begin{array}{l}\text { No se parte de un } \\
\text { diagnóstico. }\end{array}$ \\
$\begin{array}{l}\text { Desconocimiento de } \\
\text { la involucración } \\
\text { métodos pedagógicos. } \\
\text { ficación. }\end{array}$ & $\begin{array}{l}\text { e interés de los el pro- } \\
\text { grama, no el apren- } \\
\text { dizaje. }\end{array}$ \\
$\begin{array}{l}\text { Desconocimiento de } \\
\text { didácticas específicas. } \\
\text { Desconocen el modelo } \\
\text { de competencia de la } \\
\text { SEP. }\end{array}$ & $\begin{array}{l}\text { El profesor se limi- } \\
\text { ta al papel de eje- } \\
\text { cutor de cursos. }\end{array}$ & $\begin{array}{l}\text { tancia a otros fac- } \\
\text { tores que al apren- } \\
\text { dizaje }\end{array}$ & $\begin{array}{l}\text { La enseñanza es } \\
\text { episódica. }\end{array}$ \\
\end{tabular}

Fuente: Elaboración propia a partir de observaciones de clase y bitácoras de los profesores.

Con la idea de apoyar a los profesores a integrar los distintos aspectos de la práctica de la enseñanza de las matemáticas se diseñó un modelo que les permita una visión de conjunto y la relación de las partes. El modelo educativo se concibe como “... una construcción teórica, un prototipo y una representación idealizada de un proceso que describe su funcionamiento y permite la prescripción de un cauce de acción" (Díaz Barriga, 2012: 27). El modelo permitiría a los profesores articular conocimientos, técnicas y recursos con que cuenta, en una estrategia coherente. Al mismo tiempo señalaría las carencias para que los profesores busquen maneras de subsanarlas. 


\section{El modelo}

El modelo que se propone fue elaborado con base en las dificultades señaladas previamente: motivación, enseñanza y evaluación, y de una revisión cuidadosa de la literatura especializada y la investigación acerca de la enseñanza eficaz de las matemáticas en la educación secundaria llevada a cabo por expertos en matemáticas, procesos de enseñanza aprendizaje y metodología. El modelo no aborda la problemática de la primera columna de la tabla 1: el desconocimiento del modelo de competencias de la SEP, de métodos pedagógicos, de didácticas específicas, ni del contenido de la disciplina.

El modelo propuesto tiene tres dimensiones: la enseñanza de las matemáticas, el aprendizaje de los alumnos y la evaluación; además, tiene dos ejes transversales que cruzan todas las dimensiones: la idea de la matematización de la realidad; es decir, que el alumno piense su entorno en términos matemáticos, y los aprendizajes esperados que la autoridad educativa ha señalado como aquellos aspectos relevantes que integran la competencia matemática. Éstos deben de estar presentes permanentemente en la mente del profesor y los alumnos durante el proceso de enseñanzaaprendizaje. Gráficamente el modelo se representa de la siguiente manera:

Modelo para la enseñanza de las matemáticas

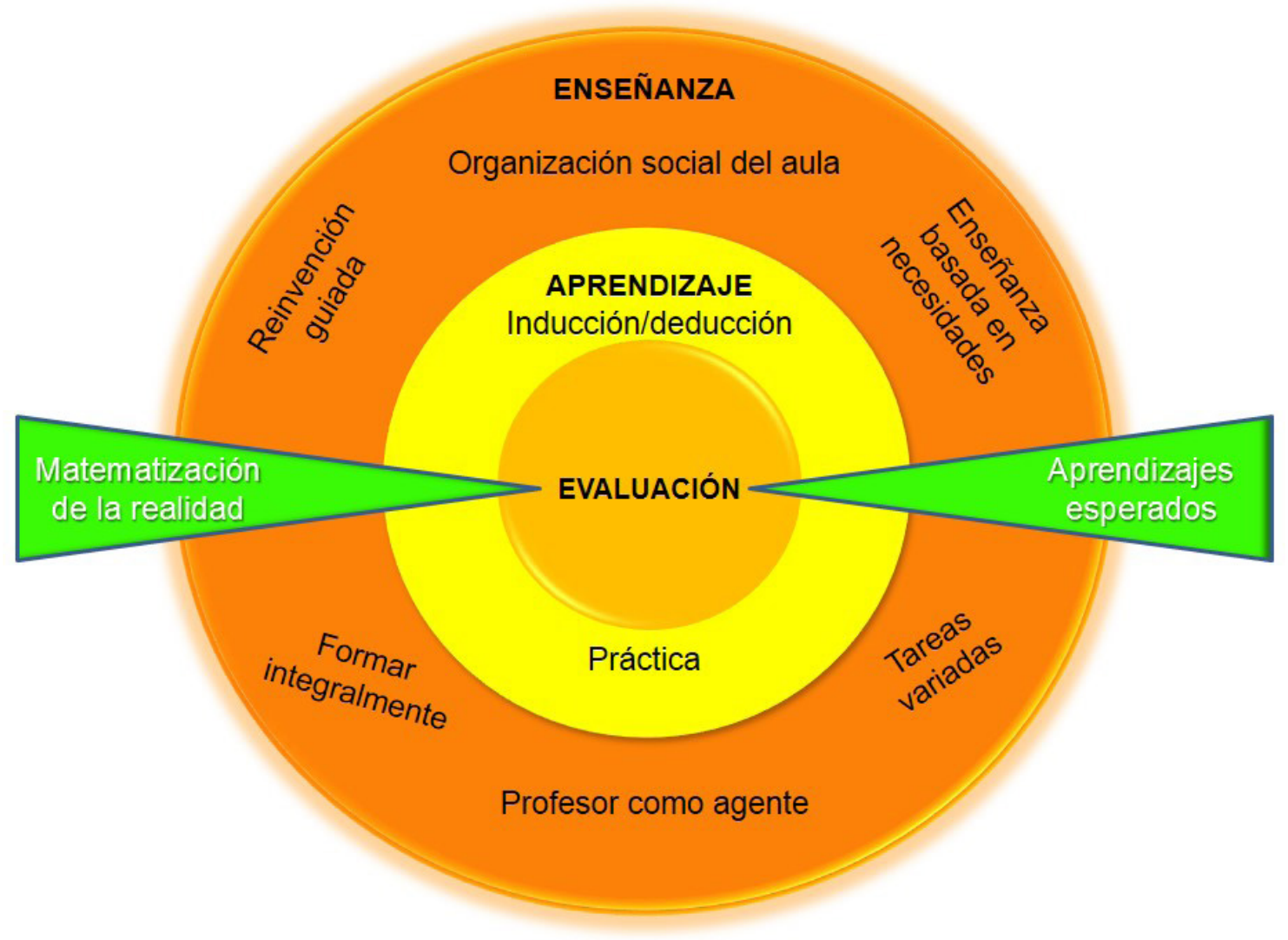

Fuente: Elaboración propia 
Integrar las dimensiones y ejes que se plantean en el modelo no es tarea fácil, por lo que en las páginas siguientes se desagregan sus componentes.

\section{Las dimensiones}

Las dimensiones son las invariables en todo proceso educativo, ocurren simultáneamente y cada uno influye en los otros, presentarlas por separado es sólo un ejercicio analítico que tiene la finalidad de comunicarlos con claridad. A continuación se presentan en el mismo orden en que aparecen en el esquema: enseñanza, aprendizaje y evaluación.

\section{Enseñanza}

La primera dimensión del modelo es la enseñanza, aunque el centro de un programa educativo sea el aprendizaje. En el término enseñanza se engloban todas aquellas acciones que realiza el profesor para que los alumnos aprendan. Los aspectos más importantes de la enseñanza son: centrarse en el aprendizaje y no en el cumplimiento del programa, que el profesor se asuma como agente educativo y no como operario, organizar socialmente el aula según la naturaleza de la tarea, enseñar basándose en las necesidades de los alumnos, guiar la reinvención de las matemáticas, incluir tareas diversas de manera simultánea y contribuir a la formación integral del alumno.

\subsection{Centrarse en el aprendizaje y no en el cumplimiento al programa}

Los profesores tienden a seguir un programa de manera rígida y es frecuente escucharlos decir que sus clases están bien porque van al día en el cumplimiento del programa. Esto significa que los profesores exponen los temas, piden al alumno que practique un poco y siguen adelante. El aprendizaje de los alumnos poco se toma en cuenta. Éste es un efecto perverso generado por la autoridad educativa que tiene todo el proceso pautado para el profesor.

Con cierta frecuencia los profesores buscan materiales educativos atractivos para los alumnos, algunos concretos y otros informáticos y refieren que eso atrae la atención de los alumnos; sin embargo, en muchos casos se centran en el medio por sí mismo y en la capacidad del medio de atraer la atención del alumno. Habría que centrarse en que los alumnos aprendan (unEsCO, 1998; Ausubel, 1989) y no en que el medio sea atractivo o en cubrir el programa.

\subsection{Que el profesor se asuma como agente educativo}

El profesor es considerado un técnico a quien se le deben dar todos los insumos: un programa, planeaciones específicas para cada día, fechas de cumplimiento, etcétera, su función es únicamente la de operar un programa que alguien más diseñó. Con esa concepción del profesor como técnico, se ha enfatizado el cumplimiento de un programa y no el aprendizaje de los estudiantes. El profesor no tiene papel de agente, sino de operario. 
El profesor no debe ser operario, es parte fundamental en el proceso de enseñanza aprendizaje como mediador ente el alumno y el objeto de conocimiento (Feuerstein, Rand, Hoffman y Miller, 1980). No puede trabajar con una recomendación específica sino que debe utilizar los distintos medios a su alcance para apoyar al alumno en su proceso de aprendizaje: materiales concretos, TIC, ejercicios, etc. Además le corresponde motivar al alumno a aprender (Willis, 2010) mostrándole la utilidad del aprendizaje y su propio interés en el conocimiento matemático.

La motivación para el aprendizaje no debe centrarse en la selección o diseño de actividades amenas, divertidas o sencillas. Una actividad de aprendizaje tiene como finalidad que los alumnos realicen esfuerzos en actividades que promuevan la curiosidad y la creatividad de modo que se impliquen en el proceso de aprendizaje esperando obtener a cambio conocimiento o competencias (Gómez, 2005) y no diversión o calificaciones.

Algunas maneras de incrementar la motivación para el aprendizaje son las siguientes:

* Graduar la dificultad de las actividades.

* Plantear las metas de aprendizaje con claridad.

* Asegurar que los alumnos tengan éxito en las tareas.

* Variar la forma y la presentación de los contenidos.

* Mostrar la utilidad del conocimiento para la vida.

* Plantear problemas interesantes.

* Ayudar a los alumnos a generalizar lo aprendido a otras situaciones.

* Aprender en colaboración con otros (Santamaría, 2006).

\subsection{Organizar socialmente el aula según la naturaleza de la tarea}

Existen algunas prácticas de aula que suelen ser señaladas como las responsables de la baja efectividad en la educación básica. Una de ellas es que se pretende lograr que los alumnos aprendan a través del discurso del profesor mientras escuchan pasivamente. También hay una crítica a un inductivismo mal entendido, en el que se pone a los alumnos a trabajar en grupo sin tener una meta, un producto, un tiempo, y una razón clara. El trabajo en grupos puede ser una pérdida de tiempo o una buena manera de promover el aprendizaje. Que sea lo uno o lo otro dependerá de la naturaleza de la tarea y del diseño de la actividad.

Tres estructuras organizativas que se pueden utilizar en el aula para fomentar el aprendizaje son: el grupo completo, el trabajo en grupos pequeños y el trabajo personal (Dubinsky, Reynolds, Schwingendorf, Vidakovic, Shahin y Winbish, 1995), no necesariamente individual. Cada una de ellas es apropiada para distintos propósitos. Por ejemplo, hay ocasiones en que es necesario hacer una introducción general al tema, demostrar algunos procedimientos, analizar el desempeño del grupo o dar instrucciones generales en donde el grupo completo es la organización social más apropiada. 
Si se quiere dar seguimiento al desempeño de cada uno de los alumnos y posibilitar que utilicen la inteligencia colectiva (Perkins, 1994) para que unos alumnos aprendan de otros mientras resuelven una tarea específica, o si se busca dar orientación e información precisa en el nivel en que cada alumno lo requiere, conviene utilizar una estructura de organización social de grupo pequeños (5 a 7 alumnos) (Dubinsky et al., 1995). En estas agrupaciones el profesor puede observar el desempeño de cada uno de los miembros y proporcionar ayuda específica en aquello que no logren resolver (Vigotsky, 1979).

En los ambientes colaborativos, los alumnos tienen la oportunidad de interactuar con sus compañeros para discutir y reflexionar acerca de la información encontrada bajo la supervisión del profesor (Dubinsky et al., 1995). Éste puede estimular a los estudiantes mediante la búsqueda guiada de información acerca de los temas de clase, presentando y recomendando lineamientos de consulta de documentos, imágenes, vídeos, etcétera.

Una estructura de organización social en que los alumnos trabajen de manera personal es conveniente cuando se trata de que desarrollen alguna habilidad específica, que ya poseen, pero que es necesario que la extiendan y refinen. Se enfatiza que es de manera personal, lo cual no significa individual. Los alumnos pueden estar trabajando por su cuenta y pedir ayuda a cualquiera de sus compañeros en aspectos muy específicos de la tarea que están realizando.

\subsection{Enseñanza basada en necesidades}

Los alumnos llegan a la secundaria con distinto nivel de conocimientos y habilidades, pero además el ritmo en que aprenden varía notoriamente de un alumno a otro, por tanto, la enseñanza genérica para todos, solamente servirá a unos cuantos alumnos. El aprendizaje no ocurre cuando el alumno puede hacer las cosas sólo, ni tampoco cuando el alumno carece de los requisitos previos para lograrlo. El aprendizaje sólo ocurre cuando el estudiante puede hacer, con la ayuda de otro, lo que solo no podía. Si se quiere que todos los alumnos aprendan habría que ayudarles en aquello que cada uno necesita.

Para poder hacer esto es necesario utilizar alguna de las estructuras de organización social que previamente se mencionaron, particularmente la de grupos pequeños en donde el profesor o un alumno más aventajado ayuda a otros que no pueden realizar la actividad de aprendizaje solos, pero sí con la ayuda de alguien más capaz (Gómez y Gómez, 2012).

\subsection{Reinvención guiada}

Buena parte de las matemáticas surgieron como respuesta a necesidades específicas en un momento histórico y en un contexto social determinado. Por ejemplo, en Egipto desarrollaron la geometría para medir los terrenos adyacentes al río Nilo, dado que éste se desbordaba todos los años y borraba los linderos. Se puede ayudar a los alumnos a comprender las matemáticas mostrándoles la necesidad que resolvían en ese contexto histórico social y geográfico en que aparecieron. Para ello se 
puede utilizar la reinvención guiada, que consiste en guiar al alumno a que descubra por sí mismo, lo que algún matemático descubrió en el pasado (Van den Heuvel-Panhuizen, 2000). Un ejemplo de esto sería llevarlos paso a paso para que reconstruyan el teorema de Pitágoras.

\subsection{Inclusión de tareas diversas y simultáneas}

La práctica común en las aulas de secundaria es que el profesor demuestre procedimientos para que el alumno los siga; sabemos que ésta no es la mejor manera de fomentar el aprendizaje de todos los alumnos. Relacionado con la organización social del aula, los profesores podrían establecer actividades simultáneas pero diferentes, apropiadas a los distintos niveles de aprendizaje de los alumnos en el aula (Tharp, Estrada, Dalton y Yamauchi, 2002). En el mismo salón, simultáneamente distintos grupos de alumnos podrían estar trabajando, algunos en la comprensión de la noción de fracción, otros en operaciones y otros en resolución de problemas.

\subsection{Formar integralmente al alumno}

El profesor no puede restringirse a la enseñanza de contenidos matemáticos. Al tiempo que hace esto debe fungir como líder que modela, crea las estructuras de convivencia que incidan en una cultura de respeto y tolerancia, solidaridad y equidad (Cava y Musitu, 2006; Ianni, 2006).

La enseñanza ocurre en un entorno social con reglas similares a las de la sociedad en general. De ahí la importancia que en el aula se fomente el respeto a las personas y a las normas que regulan la convivencia para lograr que ésta sea armónica y solidaria. El profesor debe procurar la generación de un ambiente propicio en el que se valore el esfuerzo y se reconozcan los avances de los alumnos y se exija respeto a todos los participantes del grupo.

La educación para la convivencia en las escuelas, donde el alumno habita una parte importante del día, pues la convivencia es un intento de equilibrio entre las necesidades individuales y los intereses colectivos, entre el deseo y la ley. Palabras clave de la convivencia como: comunicación, diálogo, respeto mutuo, participación, compromiso, etc., serán carentes de significado, vacías de contenido, si no se les reconoce en actos, si no se les vive (Ianni, 2006), por ello se debe fomentar la socialización que "consiste en el progresivo control de los impulsos individuales que posibilitan acceder a las interrelaciones sociales" (Ianni y Pérez, 2006: 149).

$\mathrm{Al}$ atender la convivencia durante la enseñanza hay que tomar en cuenta que los valores inherentes a las relaciones no se pueden enseñar por decreto sino que deben estar presentes en el propio clima social de la escuela, que las humillaciones y la exclusión no son naturales ni inevitables, que hay que enseñar a los alumnos formas pacíficas de resolver los conflictos y que cierto porcentaje de alumnos siempre son rechazados (Cava y Musitu, 2006). 


\section{Aprendizaje}

Respecto al aprendizaje se destacan dos aspectos: el proceso inductivo y deductivo así como la importancia de que los alumnos tengan práctica suficiente para lograr una comprensión a fondo del conocimiento matemático así como el dominio de procedimientos.

\subsection{Aprendizaje inductivo y deductivo}

El punto central es que el aprendizaje de las matemáticas en secundaria, la mayor parte del tiempo debe iniciar a través de procesos inductivos (Webb, Van Der Kooij, Henk, Geist, 2011). Por ejemplo, se puede pedir a los alumnos que midan los ángulos internos de diversos triángulos que ellos mismos elaboren, para que lleguen a una conclusión. Evidentemente, la conclusión a la que llegarán es que la suma de los ángulos internos de cualquier triángulo es de $180^{\circ}$ independientemente de su tamaño o tipo.

La inducción es una operación mental a través de la cual el aprendiz llega a una conclusión después de revisar muchos casos concretos. Otro ejemplo de actividad para el aprendizaje inductivo es que el profesor solicite a los alumnos que midan la circunferencia de un objeto redondo como un plato, luego que dibuje un punto en el centro del plato y mida el diámetro -lo que mide el plato de un lado a otro de su circunferencia pasando por su centro- enseguida dividirá la circunferencia entre el diámetro. Posteriormente se le pedirá que repita la operación con distintos objetos redondos: vasos, tapas, etcétera y el alumno llegara a la conclusión que el resultado de dividir la circunferencia entre el radio es una constante, independientemente del tamaño de los objetos (aproximadamente 3.14159265...). Así, el estudiante, inductivamente, habría descubierto el valor de $p i$ y algunas de sus características -número irracional con infinitos decimales.

Aunque se señala que el aprendizaje de las matemáticas en este nivel educativo debe ser principalmente inductivo, no se puede disociar de la operación mental inversa: el razonamiento deductivo. En el razonamiento inductivo se parte de una premisa y a partir de ella se llega a conclusiones sobre casos particulares. A partir del ejemplo de la suma de los ángulos internos de un triángulo. Se le puede pedir al alumno que encuentre la apertura de un ángulo rectángulo, en donde uno de sus ángulos es de $40^{\circ}$. En este ejemplo, el alumno parte de la premisa que la suma de los ángulos internos de cualquier tríangulo es de $180^{\circ}$. También sabe que un triángulo rectángulo tiene un ángulo recto; es decir, de $90^{\circ}$, ya se le dio el valor de otro de los ángulos $40^{\circ}$. A partir de la premisa le será fácil obtener el resultado: $90^{\circ}+40^{\circ}=130^{\circ}$. La diferencia entre $180^{\circ}$ y $130^{\circ}$ grados es $50^{\circ}$ por lo que necesariamente el tercer ángulo será de ese valor.

Otro ejemplo de deducción es el siguiente: cuando se quiere saber el costo de un producto al que no se le ha agregado el IVA (16\%), se suele multiplicar por 1.16. El resultado da el costo del producto ya con el IVA incluido. Por ejemplo, si el producto cuesta 50 'pesos, al multiplicarlo por 1.16 nos dará 58 pesos como costo total del producto. Ahora, se le puede preguntar al alumno qué hacer para obtener el costo sin IVA de un producto que lo tiene incluido. El asunto no es que el alumno 
sepa otro procedimiento, sino que lo deduzca a partir de su conocimiento previo. Si el alumno sabe que la multiplicación tiene una operación inversa que es la división; es decir, una operación que hace exactamente lo contrario de la multiplicación, sabrá que esa es la manera de revertir lo que ya hizo. Si multiplicar por 1.16 le suma el IVA al producto, la operación inversa se lo quita. Este tipo de razonamiento debe fomentarse en los alumnos de secundaria.

\subsection{Práctica}

En este modelo la práctica es un elemento central. La práctica permite generar aprendizajes importantes, entre ellos la conceptualización y el descubrimiento de relaciones, así como la extensión y refinamiento del conocimiento. Por otra parte permite que el alumno conozca todas las posibles variaciones y relaciones de un procedimiento en particular. Para ilustrarlo, se puede pensar en el ejemplo más elemental. Un niño de kinder empieza a sumar con números que no excedan de 3 . Es decir, $3+1,3+2,3+3,2+1,2+2,2+3,1+1,1+2$ y $1+3$.

Se le pide al alumno que practique día con día estas pequeñas sumas. Inicialmente lo hace moviendo fichas en donde coloca el primer sumando como un conjunto y el segundo como otro conjunto separado, y luego, siguiendo la indicación del operador "+" une los conjuntos, cuenta las fichas y obtiene el resultado, mismo que anota al lado de la operación. Después de practicar un tiempo con las fichas, el niño podría hacerlo con los dedos, lo que le resulta más práctico y rápido.

Cualquiera que ha trabajado temas matemáticos con niños pequeños, sabe que si el niño hace la misma operación una y otra vez, vuelve a hacer el conteo, a pesar de que el adulto le diga que si tiene los mismos sumando obtendrá el mismo resultado. Al niño esta declaración del adulto no le significa nada; sin embargo, cuando él lo descubre, deja de hacer el conteo y copia el resultado de las otras sumas iguales.

A pesar de saber que si suma $3+2$, obtendrá como resultado 5 , cuando se le presenta la suma $2+3$, tiene que volver a contar, aunque se le informe que dado que si son los mismos números obtendrá el mismo resultado aún si los sumandos no están en el mismo orden. Después de un tiempo, el niño descubre que el orden en que aparecen los sumandos no altera el resultado, entonces cuando ya sumó $3+2$ y a continuación se le presenta $2+3$, ya no vuelve a sumar, sino que únicamente copia el resultado.

En este momento el adulto le podría decir que no necesita poner en sus dedos un dos y un tres, que simplemente ponga con sus dedos uno de los sumandos para agregarlo al otro. De nuevo, el niño no entiende y se rehúsa a hacerlo hasta que descubre por sí mismo, después de ejecutar la operación muchas veces que sólo necesita agregar el segundo sumando el primero.

A partir de ese momento el proceso se agiliza. Algunos niños que ya comprendieron que no importa el orden de los sumandos, representan con sus dedos invariablemente el número más pequeño, dado que así, el procedimiento es más rápido y sencillo. Finalmente, el niño memoriza los resultados de la suma. 
Valga la simplicidad del ejemplo anterior para mostrar como la práctica es un elemento fundamental en el aprendizaje de las matemáticas. Está lejos de ser un asunto mecánico, es la manera de que los alumnos descubren relaciones, desarrollan esquemas operativos, abrevian procedimientos, conocen todas las variaciones del tema que estudian y eficientizan su trabajo. Se eligió una tarea muy simple, que no corresponde al nivel de secundaria para mostrar cómo hasta en ésta puede notarse que la práctica es indispensable. En la medida en que el conocimiento es más complejo, la práctica adquiere mayor relevancia.

En el nivel de secundaria, un ejemplo sería el orden de operaciones. Si los alumnos tienen suficiente práctica, podrán comprender mejor y evitarán errores frecuentes como sumar antes de multiplicar o dividir o empezar por las operaciones que están fuera de los paréntesis.

Cuando un estudiante puede realizar ciertos procedimientos matemáticos de manera automatizada, libera recursos cognitivos que podrán ser utilizados para comprender temas más complejos; mientras que los estudiantes que no tienen procedimientos automatizados utilizarán recursos cognitivos para pensar cómo realizar los procedimientos más básicos (Andrade-Lotero, 2012).

Para que el estudiante tenga un aprendizaje eficaz, éste debe tener dominio de las operaciones básicas que se logra mediante la ejercitación, así como la capacidad de comprender los conceptos matemáticos. Se sabe que si el alumno ejercita suficientemente los aspectos operacionales de un objeto matemático, llega a ser capaz de entender la lógica subyacente y por tanto logra una comprensión abstracta que le permita abordar con autonomía problemas más complejos. Los aspectos

mecánicos y comprensivos están indisolublemente unidos y es difícil establecer una línea divisoria entre ellos.

\section{Evaluación}

En las escuelas la evaluación tiende a ser utilizada como calificación. Esa calificación que incluyen la asistencia, la participación, las tareas, la conducta y el aprendizaje no representa de manera alguna el aprendizaje de los alumnos. En ese sentido, no sirve como retroalimentación. La evaluación es "una actividad compleja, intencional y fundamentada, que pretende valorar la calidad de un proceso y/o un resultado, con la finalidad de informar del mismo para propiciar futuras decisiones justificadas que contribuyan a su mejora" Medina Rivilla (1991: 52).

Como se desprende de la definición anterior, la evaluación permite, diagnosticar el nivel de aprendizaje de los alumnos que debe de servir principalmente para retroalimentar a los alumnos sobre su desempeño y para planear o replantear nuevas situaciones de aprendizaje que lleven a cada alumno hacia el cumplimiento los aprendizajes esperados que la autoridad educativa ha señalado (Bonvechio y Maggioni, 2006).

Esta evaluación debe ser recurrente y en espiral. Debe incluir no solamente lo aprendido en el último bimestre si no los conocimientos previos que son requisito para seguir aprendiendo los nuevos temas. En forma de espiral ascendente quiere decir que primero son pocos los conocimientos 
que se evalúan pero cada vez se va ampliando ese rango de conocimientos. No se quiere decir que se deben hacer evaluaciones muy extensas, pues frecuentemente un desempeño incluye el dominio de conocimientos previos (Acevedo, 2003; Ahumada, 2005).

\section{Los ejes transversales}

El modelo, además de los tres elementos centrales contiene dos ejes transversales que cruzan todo el proceso de enseñanza aprendizaje y evaluación. Estos ejes son: la matematización de la realidad y los aprendizajes esperados.

\section{A. Matematización de la realidad}

Para que las matemáticas cobren sentido es importante que el alumno parta de situaciones concretas de la realidad y que las piense en términos matemáticos, procedimiento al que se le puede llamar matematización de la realidad (Webb et al. 2011). Por ejemplo, el alumno puede pensar en su escuela en términos matemáticos: ¿cuál es la edad promedio de los alumnos?, ¿cuál es la edad promedio de los profesores?, ¿cuánto cuesta la educación de cada alumno si se reparten los costos de los salarios de los profesores, el mantenimiento de la escuela y la renta del edificio?, ¿cuantos metros cuadrados de terreno hay por cada persona de un turno escolar? La matematización de la realidad incluye trabajar una situación concreta para la cual se hace un modelo que posteriormente se utiliza a otros casos particulares (Santamaría, 2006).

Los profesores tienen la tendencia a enseñar de manera abstracta, cuando las comprensiones más importantes ocurren en lo concreto, en la riqueza de experiencias que van a permitir que los alumnos logren comprender la estructura de las nociones matemáticas, (Dubinsky y Mc Donald, 2001), por eso el centro está en que el alumno aprenda, no en que el profesor explique. La explicación debe estar en función de la necesidad del alumno y no como el centro de la actividad.

En un ejemplo concreto, para que los alumnos puedan comprender el tema de fracciones es necesario, que se parta del conocimiento informal que poseen los alumnos, como la experiencia de dividir enteros en partes iguales, de fraccionarlos; manzanas, galletas etcétera.

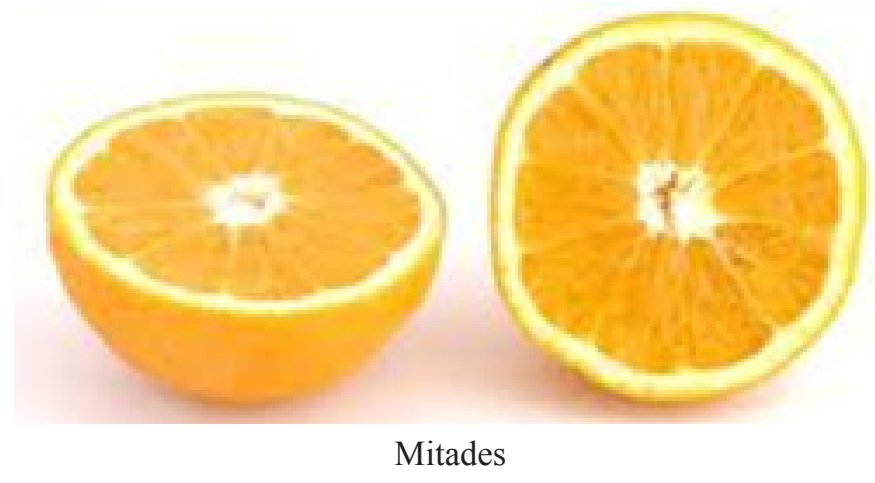

Es importante que los alumnos comprendan que un entero no necesariamente es una unidad perceptible como una naranja, un pastel, una casa, etc., sino que, además de esas, también puede 
ser cualquier conjunto que arbitrariamente deseemos considerar unidad, como una cantidad, por ejemplo 63 pesos, que aunque gráficamente no parecen unidad, lo son porque así se ha decidido considerarlos y que también puede ser convertido en fracciones.
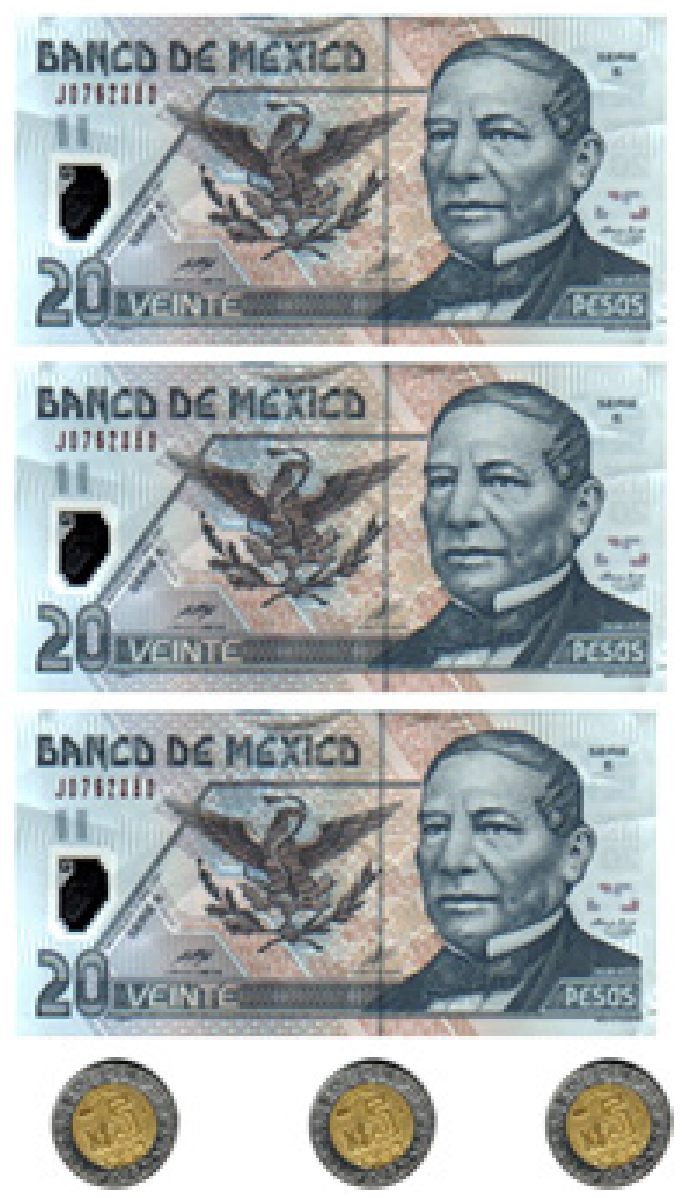

Entero, 63 pesos

Si la alumna piensa en esa unidad, 63 pesos, como una cantidad que le dieron para ella y sus dos hermanos, podría hacerla partes iguales: tres tercios, por lo que a simple vista podría darse cuenta que cada tercio consiste en 21 pesos.

Con esta variedad de experiencias los alumnos llegan a comprender la noción de entero más allá de las totalidades perceptuales. Una vez que los alumnos comprendieron la noción de número entero y números fraccionarios pueden aprender la notación convencional y posteriormente a hacer operaciones con ellos.

El punto importante es que el alumno reconozca los aspectos matemáticos de la realidad en que vive, que los conceptualice, que los represente de manera abstracta y que posteriormente apliqué ese conocimiento a nuevos aspectos concretos de la realidad. 


\section{B. Los aprendizajes esperados}

El segundo eje transversal del modelo se refiere a los aprendizajes esperados que ha planteado la autoridad educativa (SEP, 2011). Éste es el aspecto fundamental que ha de tener en mente el profesor al planear una actividad de aprendizaje y que también deberán de tener en mente él y los alumnos al momento de llevarla a cabo en el aula, al elaborar todo tipo de trabajo y durante la evaluación. Las actividades por sí mismas tienen poco valor, ya que éste reside en su capacidad para ayudar a que el alumno logre los aprendizajes que se esperan.

En las clases observadas, se notó que los profesores tienen en cuenta el temario dado que su actividad está centrada en desarrollar procedimientos y explicar conceptos a los alumnos y no en que éstos construyan el conocimiento que se espera según el grado escolar.

\section{Uso del modelo}

El profesor puede iniciar por planear la enseñanza a partir de las competencias matemáticas que señala la autoridad educativa para la educación básica, identificando los aprendizajes esperados para una unidad de tiempo de ese grado escolar y diseñando las situaciones de aprendizaje en que participarán los alumnos para lograr los aprendizajes del contenido en un ambiente de cordialidad y respeto.

Durante las clases los estudiantes construirán el conocimiento a partir de las situaciones de aprendizaje inductivas, deductivas, de reconstrucción y de descubrimiento que el profesor haya preparado, de las conceptualizaciones que éste le presente y de las actividades de práctica y repaso que les provea.

El profesor evaluará si el alumno logró los aprendizajes esperados y utilizará este conocimiento para adecuar la enseñanza a las necesidades de los estudiantes. Las dimensiones de enseñanza, aprendizaje y evaluación se distinguen por cuestión analítica pero el profesor las aborda conjuntamente. Se trata de que el alumno aprenda y para ello es necesaria la enseñanza y la evaluación. Esta última puede ser a través de actividades que son simultáneamente de aprendizaje y evaluativas.

Los ejes que atraviesan las dimensiones: matematización de la realidad y aprendizajes esperados cruzan las tres dimensiones como elementos que el docente debe tener en mente de manera permanente porque estos son los objetivos de su actividad.

\section{Consideraciones finales}

Un modelo es un esquema que representa, de manera verbal o gráfica, un proceso complejo de la realidad, pero de manera más sencilla. Por tanto, implica una presentación selectiva de aquellos aspectos de la realidad que los creadores del modelo desean destacar. Esa naturaleza representacional y selectiva de un modelo es a la vez ventaja y limitación. La ventaja reside en que permite enfocarse en los aspectos relevantes sin perderse en los detalles; la limitación es que puede dejar fuera algunos aspectos importantes. 
Para la elaboración del modelo presentado se partió de las limitaciones identificadas en prácticas educativas concretas y el conocimiento especializado sobre el tema. A pesar de estar anclado en la realidad, podría no responder a todas las necesidades; sin embargo, presenta una estructura coherente para abordar la práctica de la enseñanza de las matemáticas.

Los profesores de secundaria podrían utilizar este modelo para contrastar su práctica con lo que se propone y modificar, si fuera pertinente, aquellas acciones que no conducen a un mejora del aprendizaje, si son escépticos, podrían ponerlo a prueba y determinar si esta propuesta les da mejores resultados que lo que actualmente hacen; también podrá servir para tener un marco de referencia para observar y retroalimentar clases de matemáticas en el nivel de secundaria.

Una comunidad de práctica sería escenario apropiado para analizar y discutir el modelo, para hacerle modificaciones y darle un sentido compartido. En ese mismo espacio colegiado se podría evaluar su utilidad como marco para planear, implementar acciones pedagógicas y evaluar tanto los procesos de enseñanza como el logro de los aprendizajes esperados.

\section{Referencias}

Acevedo, Myriam. (2003). "La evaluación en el aula de matemáticas”. En Bogoya, Daniel (ed.).

Trazas y Miradas, evaluación y competencias. Bogotá: Universidad Nacional de Colombia.

Ahumada, Pedro. (2005). Hacia una evaluación auténtica del aprendizaje. México: Paidós.

Andrade-Lotero, L. (2012). "Teoría de la carga cognitiva, diseño multimedia y aprendizaje: un estado del arte”. Magis: Revista Internacional ee Investigación en Educación, 5(10), pp. 75-92. Ausubel, D. (1989). Psicología Educativa. Un punto de vista cognoscitivo. México: Trillas.

Bonvecchio, Mirta y Beatriz Maggioni. (2006). Evaluación de los aprendizajes. Buenos Aires: Novedades Educativas.

Cava, María de Jesús y Gonzalo Musitu. (2006). "El porqué y el cómo del programa 'convivir"”. En Luna, María Eugenia, Martha Tortolero, Antonio V. y José Sánchez (comps.). Orientación y Tutoría. Antologia, Primer taller de Actualización sobre los Programas de Estudio 2006. Reforma de la Educación Secundaria. México, SEP, pp. 113-129.

Díaz Barriga, F. (2012). "Reformas curriculares y cambio sistémico: Una articulación ausente pero necesaria para dar cabida a la innovación". Revista Iberoamericana de Educación Superior (RIES), 3 (7), 23-40.

Dubinsky E.; N. Hagelgans; B. Reynolds; K. Schwingendorf; D. Vidakovic; M. Shahin y J. Wimbish. (1995). "A Practical Guide to Cooperative Learning in Collegiate Mathematics". Notes 37, Washington: MAA.

Dubinsky E. y M. Mc Donald (2001). "APOS: A Constructivist Theory of Learning in Undergrad Mathematics Education Research, 2012". En Holton, D. (ed.). The teaching and Learning of Mathematics at University Level: An ICMI Study. Kluwer Academic Publishers, pp. 273-280.

Feuerstein, R.; Y. Rand; M. B. Hoffman y R. Miller. (1980). Instrumental Enrichment: an intervention program for cognitive modifiability. Baltimore: University Park Press. 
Gómez, Inés M. (2005). "Motivar a los alumnos de secundaria para hacer Matemáticas”. Recuperado de http://www.mat.ucm.es/ imgomezc/almacen/pisa-motivar, el 21 de febrero de 2014.

Gómez, L. F. y A. Gómez (2012). "El aprendizaje mediado entre compañeros para el manejo de programas computacionales". En Sinéctica, 38, enero-junio, 2012. Recuperado de http://www. sinectica.iteso.mx/index.php?cur=38\&art=38 02. ISNN:1665-109X

Ianni, Norberto. (2006). "La convivencia escolar: una tarea necesaria, posible y compleja". En Luna, María Eugenia, Martha Tortolero, Antonio V. y José Sánchez (comps.). Orientación y Tutoría. Antología, Primer taller de Actualización sobre los Programas de Estudio 2006. Reforma de la Educación Secundaria. México: SEP, pp. 177-191.

y Elena Pérez. (2006). "Convivencia, conflictos y sanciones". En: Luna, María Eugenia, Martha Tortolero, Antonio V. y José Sánchez (comps.). Orientación y Tutoría. Antología, Primer taller de Actualización sobre los Programas de Estudio 2006. Reforma de la Educación Secundaria. México: SEP, pp. 131-151.

INEE (2012). Estudio comparativo del aprendizaje en tercero de secundaria en México 2005-2008. Español y Matemáticas. México: Autor.

Libâneo, J. (2014). "La integración entre el conocimiento disciplinar y el conocimiento pedagógico en la formación de Profesores y la contribución de la teoría de la enseñanza de Vasili Davídov". Revista de Didácticas Especificas, núm. 10, pp. 5-37.

Mathematics Education in the Netherlands: A guided tour. Freudenthal Institute Cd-rom for ICME9. Utrecht: Utrecht University.

Medina Rivilla, A. (1991). Teoría y Métodos de Evaluación. Madrid: CINCEL.

OCDE (2013). OECD. Recuperado de http://www.oecd.org/pisa/aboutpisa/PISA\%20scales\%20 for\%20pisa-based\%20test $\% 20$ for\%20schools.pdf, el 5 de Diciembre de 2013

Perkins, D. (1994). "Where is the intelligence?". Educational Leadership, pp. 105-106.

Rodríguez, R. y E. Zuazua. (2002). "Enseñar y aprender Matemáticas: del Instituto a la Universidad". Recuperado de http://eprints.ucm.es/9538/1/enseniaryaprender.pdf, el 20 de febrero de 2014.

Santamaría, F. (2006). La contextualización de la Matemática en la escuela primaria de Holanda. Tesis de Maestría en Enseñanza de las Ciencias Exactas y Naturales, con orientación en Matemática. Recuperado de http://www.gpdmatematica.org.ar/publicaciones/tesis \%20final_santamaria/1.pdf, el 20 de febrero del 2014.

SEP (2011b). Programas de estudio 2011. Guía para el maestro. Educación Básica Secundaria. Matemáticas. México: SEP..

Smeby, J. (1996). "Disciplinary Differences in University Teaching”. Studies in Higher Education. Vol. 21, núm. 1, pp. 69-69.

Tharp, R.; P. Estrada; S. Dalton y L. Yamauchi. (2002). Transformar la enseñanza: excelencia, equidad, inclusión y armonía en las aulas y las escuelas. España: Paidós. 
UNESCO (1998). “Conferencia Mundial sobre Educación Superior”. Recuperado de http://unesdoc. unesco.org/images/0011/001163/116345s.pdf.

Vygotsky, L. S. (1979). El desarrollo de los procesos psicológicos superiores. Buenos Aires: Grijalbo.

Webb, David; Kooij Van der y Geist Henk (2011). "Design Research in the Netherlands: Introducing Logarithms Using Realistic Mathematics Education". Journal of Mathematics Education at Teachers College. Spring-summer 2011, Vol. 2.

Willis, J. (2010). Learning to love math. Teaching strategies that change student attitudes and get results. Alexandria, VA: ASCD.

Recibido: 28/11/14

Dictaminado: 19/04/15

Corregido: 21/04/15

Aceptado: 07/05/15 